\title{
2P/Encke, the Taurid complex NEOs and the Maribo and Sutter's Mill meteorites ${ }^{\star}$
}

\author{
C. Tubiana ${ }^{1}$, C. Snodgrass ${ }^{2,1}$, R. Michelsen ${ }^{3}$, H. Haack ${ }^{3}$, H. Böhnhardt ${ }^{1}$, A. Fitzsimmons ${ }^{4}$, and I. P. Williams ${ }^{5}$ \\ 1 Max Planck Institute for Solar System Research, Justus-von-Liebig-Weg 3, 33077 Göttingen, Germany \\ e-mail: tubiana@mps.mpg.de \\ 2 Planetary and Space Sciences, Department of Physical Sciences, The Open University, Milton Keynes, MK7 6AA, UK \\ ${ }^{3}$ Centre for Star and Planet Formation, Natural History Museum of Denmark, University of Copenhagen, 1350 Copenhagen, \\ Denmark \\ 4 Astrophysics Research Centre, Department of Physics and Astronomy, Queen's University Belfast, Belfast BT7 1NN, UK \\ 5 School of Physics and Astronomy, Queen Mary, University of London, London E1 4NS, UK
}

Received 12 December 2014 / Accepted 29 September 2015

\begin{abstract}
Aims. 2P/Encke is a short period comet that was discovered in 1786 and has been extensively observed and studied for more than 200 years. The Taurid meteoroid stream has long been linked with $2 \mathrm{P} /$ Encke owing to a good match of their orbital elements, even though the comet's activity is not strong enough to explain the number of observed meteors. Various small near-Earth objects (NEOs) have been discovered with orbits that can be linked to 2P/Encke and the Taurid meteoroid stream. Maribo and Sutter's Mill are CM type carbonaceous chondrite that fell in Denmark on January 17, 2009 and April 22, 2012, respectively. Their pre-atmospheric orbits place them in the middle of the Taurid meteoroid stream, which raises the intriguing possibility that comet $2 \mathrm{P} /$ Encke could be the parent body of CM chondrites.

Methods. To investigate whether a relationship between comet 2P/Encke, the Taurid complex associated NEOs, and CM chondrites exists, we performed photometric and spectroscopic studies of these objects in the visible wavelength range. We observed 2P/Encke and 10 NEOs on August 2, 2011 with the FORS instrument at the $8.2 \mathrm{~m}$ Very Large Telescope on Cerro Paranal (Chile).

Results. Images in the R filter, used to investigate the possible presence of cometary activity around the nucleus of $2 \mathrm{P} /$ Encke and the NEOs, show that no resolved coma is present. None of the FORS spectra show the $700 \mathrm{~nm}$ absorption feature due to hydrated minerals that is seen in the CM chondrite meteorites. All objects show featureless spectra with moderate reddening slopes at $\lambda<800 \mathrm{~nm}$. Apart for $2003 \mathrm{QC}_{10}$ and $1999 \mathrm{VT}_{25}$, which show a flatter spectrum, the spectral slope of the observed NEOs is compatible with that of $2 \mathrm{P} /$ Encke. However, most of the NEOs show evidence of a silicate absorption in lower $\mathrm{S} / \mathrm{N}$ data at $\lambda>800 \mathrm{~nm}$, which is not seen in 2P/Encke, which suggests that they are not related.

Conclusions. Despite similar orbits, we find no spectroscopic evidence for a link between 2P/Encke, the Taurid complex NEOs and the Maribo and Sutter's Mill meteorites. However, we cannot rule out a connection to the meteorites either, as the spectral differences may be caused by secondary alteration of the surfaces of the NEOs.
\end{abstract}

Key words. comets: general - comets: individual: 2P/Encke - minor planets, asteroids: general

\section{Introduction}

Comet 2P/Encke is a short-period comet that was discovered in 1786 and it has been studied for more than 200 years. It has an orbital period of 3.3 years and its orbit is dynamically decoupled from Jupiter's control because of gravitational interaction with terrestrial planets (Levison et al. 2006). It is the only comet known to be on such an orbit (107P/Wilson-Harrington has a similar orbit, but its status as an active comet is questionable, because it does not show repetitive activity each orbit). The lack of close encounter with Jupiter results in a very smooth orbital evolution (Levison et al. 2006) but raises an interesting problem relating to the origin of this comet, since such encounters are essential if it is to be captured from the outer solar system. A slow orbital evolution onto its present orbit is also unlikely since all the volatiles would have been exhausted. There are two

* Based on observations performed at the European Southern Observatory, Paranal, Chile: Program 087.C-0788(A). interesting possibilities to explain this: Either comet 2P/Encke has an origin within the main asteroid belt - recent discoveries (Hsieh \& Jewitt 2006; Jewitt 2012) re-enforce this possibility or there was a major fragmentation of an earlier larger comet that led to significant changes in the aphelion distance of the resulting fragments (Whipple 1940).

The nucleus of $2 \mathrm{P} /$ Encke is dark (geometric albedo of $0.047 \pm 0.023$ (Fernández et al. 2000)), has an effective radius of $2.4 \pm 0.3 \mathrm{~km}$ (Fernández et al. 2000), and it has polarimetric properties that are unique compared to other measured types of solar system objects, such as asteroids, TNOs, cometary dust, and Centaurs (Böhnhardt et al. 2008). The colours of 2P/Encke's nucleus are typical for comets (Jewitt 2002; Snodgrass et al. 2006), and a noisy spectrum reveals a moderate red slope (Luu \& Jewitt 1990).

The Taurid meteoroid stream has long been associated with $2 \mathrm{P} /$ Encke because of the similarity in their orbital elements. However the activity of $2 \mathrm{P} /$ Encke at the present time is not strong enough to explain the high number of meteoroids in the 
Table 1. Observational circumstances.

\begin{tabular}{lccccccccc}
\hline \hline & & \multicolumn{7}{c}{ Photometry } & Spectroscopy \\
Object name & $r^{a}(\mathrm{AU})$ & $\Delta^{b}(\mathrm{AU})$ & $\alpha^{c}\left({ }^{\circ}\right)$ & $t(\mathrm{~s}) \times N^{d}$ & $t(\mathrm{~s}) \times N^{e}$ & $e^{f}\left({ }^{\circ}\right)$ & $i^{g}\left({ }^{\circ}\right)$ & $a^{h}(\mathrm{AU})$ & $q^{i}(\mathrm{AU})$ \\
\hline $2 \mathrm{P} /$ Encke & 3.65 & 2.71 & 6.98 & $180 \times 3$ & $360 \times 3$ & 0.85 & 11.78 & 2.21 & 0.34 \\
\hline $1998 \mathrm{QS}_{52}$ & 2.90 & 2.19 & 16.44 & $180 \times 5$ & $180 \times 4$ & 0.86 & 17.56 & 2.20 & 0.31 \\
$1999 \mathrm{RK}_{45}$ & 2.11 & 1.20 & 16.57 & $180 \times 4+60 \times 3$ & $1200 \times 3$ & 0.77 & 5.89 & 1.60 & 0.36 \\
$2003 \mathrm{QC}_{10}$ & 1.52 & 0.78 & 37.64 & $180 \times 4$ & $210 \times 4$ & 0.73 & 5.04 & 1.37 & 0.37 \\
$2003 \mathrm{UL}_{3}$ & 3.10 & 2.12 & 6.55 & $180 \times 4+60 \times 6$ & $1200 \times 3$ & 0.80 & 14.66 & 2.25 & 0.46 \\
\hline $1999 \mathrm{VT}_{25}$ & 1.14 & 0.23 & 52.85 & $11 \times 5+5 \times 5$ & $200 \times 3$ & 0.52 & 5.15 & 1.16 & 0.55 \\
$2001 \mathrm{VH}_{75}$ & 1.63 & 0.84 & 31.16 & $60 \times 3+20 \times 2$ & $200 \times 3$ & 0.74 & 10.62 & 2.10 & 0.55 \\
$1984 \mathrm{~KB}$ & 3.32 & 2.31 & 1.83 & $120 \times 3+20 \times 2$ & $200 \times 3$ & 0.76 & 4.85 & 2.22 & 0.53 \\
$1998 \mathrm{SS}_{49}$ & 2.71 & 2.03 & 18.56 & $180 \times 4+20 \times 1$ & $380 \times 3$ & 0.64 & 10.76 & 2.37 & 0.69 \\
$2004 \mathrm{WS}_{2}$ & 1.54 & 0.85 & 38.14 & $180 \times 3$ & $200 \times 3$ & 0.60 & 8.26 & 1.34 & 0.53 \\
$2002 \mathrm{SY}_{50}$ & 1.65 & 1.30 & 37.93 & $180 \times 4+30 \times 1$ & $200 \times 4$ & 0.69 & 8.75 & 1.71 & 0.53 \\
\hline $2000 \mathrm{CT}_{33}$ & 2.78 & 1.82 & 8.10 & $60 \times 3+10 \times 2$ & $70 \times 3$ & 0.03 & 1.28 & 2.83 & 2.76 \\
$2004 \mathrm{RP}_{191}$ & 2.11 & 1.14 & 10.94 & $60 \times 3+10 \times 2$ & $70 \times 3$ & 0.13 & 6.23 & 2.39 & 2.08 \\
\hline
\end{tabular}

Notes. ${ }^{(a)}$ Heliocentric distance (AU). ${ }^{(b)}$ Geocentric distance (AU). ${ }^{(c)}$ Phase angle $\left({ }^{\circ}\right) .{ }^{(d)}$ Photometry: Exposure time (s) x Number of exposures. ${ }^{(e)}$ Spectroscopy: Exposure time (s) x Number of exposures. ${ }^{(f)}$ Eccentricity. ${ }^{(g)}$ Inclination. ${ }^{(h)}$ Semi-major axis. ${ }^{(i)}$ Perihelion distance.

stream. Whipple (1940) suggested that several thousand years ago a giant comet fragmented that resulted in $2 \mathrm{P} /$ Encke and several other bodies within the meteoroid complex, as well as a large number of meteoroids. This general hypothesis is supported by the fact that numerous authors agree (see Jopek \& Williams 2013 for a list) that the stream is, in fact, a complex of several smaller meteoroid streams and filaments. The most recent list of such sub-streams is given by Porubčan et al. (2006). Further support for this hypothesis came when Clube \& Napier (1984) showed that several Apollo asteroids had orbits that were very similar to the Taurid stream while a decade later, Asher et al. (1993) suggested that the complex of meteoroid streams, comet 2P/Encke, and the then known associated Apollo asteroids, could all have been formed by the fragmentation of a giant comet 20 to $30 \mathrm{ky}$ ago. Since that date several other authors have claimed associations between NEOs and the Taurid complex, the present number of candidates being well in excess of 100. Orbital evolution is fairly rapid in this locality of the solar system and most asteroids have low inclination, many of the supposed orbital similarities are fortuitous, with orbits being similar at the present time, but implying no generic connections. Porubčan et al. (2004) argue that, to claim any generic relationships, the orbits of the NEOs and those of the meteoroid streams need to have remained similar for a long time, which they chose to be 5000 years. Porubčan et al. (2006) applied this criterion to the Taurids, resulting in the number of associated NEOs dropping from 100 to around 10.

All the claimed associations between comet 2P/Encke, the Taurid meteoroid stream, and some NEOs are based on similarities of their orbits. In addition to dynamical properties, common taxonomic properties can also provide an indication of a common origin for small bodies in the solar system. Taxonomic properties are poorly-known for cometary nuclei, and only few comets have spectroscopic measurements in the visible wavelength range. The existing spectra of bare nuclei are generally featureless and display different reddening slopes (Lamy et al. 2004). Given the poor $\mathrm{S} / \mathrm{N}$ ratio that is usually obtained in observations, more subtle features, such as ones from hydrated minerals, are beyond the detection limit in most cases. Multi-colour photometry is available for a few more nuclei and is all consistent with featureless red slopes (Snodgrass et al. 2006; Lamy \& Toth 2009).

If the Taurid complex NEOs are fragments of the same body as $2 \mathrm{P} /$ Encke, we expect them to have the same spectral properties as the comet nucleus. Furthermore, it would be reasonable to expect that these NEOs could show cometary activity. Recently, Popescu et al. $(2011,2014)$ published near infrared spectra of NEOs, including some associated with the Taurid complex, with the goal of testing to see if proposed members have comet-like spectral types and to compare the resulting spectra with meteorite types. While limited to only the larger proposed members, these studies found that a majority of the NEOs sampled had S-complex spectroscopic classification and were best matched by ordinary chondrite meteorite samples.

Maribo and Sutter's Mill are CM chondrites that fell on January 17, 2009 (Haack et al. 2012) and on April 22, 2012 (Jenniskens et al. 2012b). The pre-atmospheric orbits of the two meteorites are very similar and places them right in the middle of the Taurid meteoroid stream (Haack et al. 2011; Jenniskens et al. 2012b). This raises the intriguing possibility that comet $2 \mathrm{P} /$ Encke could be the parent body of $\mathrm{CM}$ chondrites, meaning that these meteorites are potentially samples of cometary material we can study in the laboratory. CM chondrites show signs of extensive aqueous alteration, which suggest that the parent body was an icy body that was at least partially molten at some point. It is therefore possible that the parent body of the CM chondrites is a comet (Lodders \& Osborne 1999). However, Jenniskens et al. (2012b) argued that CM chondrites are more likely to come from main belt asteroids since there is no evidence of aqueous alteration in Jupiter family comets.

To investigate whether a relationship between comet 2P/Encke, the Taurid complex associated NEOs and CM chondrites exists, we performed spectroscopic studies of these objects. By observing in the visible with an $8 \mathrm{~m}$ class telescope we could sample fainter objects within the Taurid complex, and observe the comet nucleus directly, adding to the work on brighter objects by Popescu et al. (2014). We also perform a direct search for cometary activity around the NEOs.

\section{Observations and data reduction}

We observed 2P/Encke and 10 NEOs in the visible wavelength range on August 2, 2011 with the FORS instrument at the $8.2 \mathrm{~m}$ Very Large Telescope on Cerro Paranal (Chile). The observational circumstances are summarised in Table 1. FORS $^{1}$

1 For more details about FORS see: http://www.eso.org/sci/ facilities/paranal/instruments/fors.html 
Table 2. Asteroids observed.

\begin{tabular}{lll}
\hline \hline Designation & Number & Name \\
\hline $1998 \mathrm{QS}_{52}$ & 16960 & - \\
$1999 \mathrm{RK}_{45}$ & 162195 & - \\
$2003 \mathrm{QC}_{10}$ & 405212 & - \\
$2003 \mathrm{UL}_{3}$ & 380455 & - \\
\hline $1999 \mathrm{VT}_{25}$ & 152828 & - \\
$2001 \mathrm{VH}_{75}$ & 153792 & - \\
$1984 \mathrm{~KB}$ & 6063 & Jason \\
$1998 \mathrm{SS}_{49}$ & 85713 & - \\
$2004 \mathrm{WS}_{2}$ & 170903 & - \\
$2002 \mathrm{SY}_{50}$ & 154276 & - \\
\hline $2000 \mathrm{CT}_{33}$ & 16204 & - \\
$2004 \mathrm{RP}_{191}$ & 198008 & - \\
\hline
\end{tabular}

(Appenzeller et al. 1998) is the visual and near UV Focal Reducer and low-dispersion Spectrograph for the VLT. Its detector consists of a mosaic of two $2 \mathrm{k} \times 4 \mathrm{k}$ MIT CCDs $(15 \mu \mathrm{m})$. In the standard resolution mode, which we used for the observations, the image scale is $0.25^{\prime \prime} /$ pix, providing a field of view of $6.8^{\prime} \times 6.8^{\prime}$. The observations were performed with the telescope tracking at the proper motion rate of the objects.

The NEOs to be observed were selected in the following way.

1. A list was compiled of all the NEOs that have present day osculating orbits that have been shown by other authors to be similar to the mean orbit of the Taurids using one of the standard test listed in Jopek \& Williams (2013).

2. This list was reduced by requiring that the NEOs were observable during the period of our observing run. With one exception, we also confined selection to those that have been numbered and so have secure orbits.

3. From this reduced list, those NEOs that have been claimed by other authors to satisfy the criterion of Porubčan et al. (2004), namely that the orbits have remained similar for 5000 years, were selected. These were $1998 \mathrm{QS}_{52}$, $1999 \mathrm{RK}_{45}, 2003 \mathrm{QC}_{10}$, and $2003 \mathrm{UL}_{3}$. We refer to these NEOs as "Group 1" objects.

4. There was telescope time available to observe more NEOs than these 4 and so some were selected from those satisfying condition 2 above that have been officially numbered and so have secure orbits. These were $1984 \mathrm{~KB}, 1998 \mathrm{SS}_{49}$, $1999 \mathrm{VT}_{25}, 2001 \mathrm{VH}_{75}, 2002 \mathrm{SY}_{50}$, and $2004 \mathrm{WS}_{2}$. We refer to these NEOs as "Group 2" objects.

In addition we observed two main belt asteroids $\left(2000 \mathrm{CT}_{33}\right.$ and $\left.2004 \mathrm{RP}_{191}\right)$ that are not related to $2 \mathrm{P} /$ Encke based on their orbits (see orbital elements in Table 1). Numbers and names, where they exist, are given for each asteroid in Table 2, but provisional designations are used throughout this paper.

\subsection{Photometry}

We observed 2P/Encke and the NEOs using the $R$ Special filter $\left(\lambda_{\text {central }}=638 \mathrm{~nm}\right)$. The exposure times and the number of exposures per object are summarised in Table 1. Bias, flat fields and photometric standard star fields were taken during the night. The images were reduced using standard techniques of bias subtraction, flat field correction, exposure time normalisation and sky background subtraction. For the flux calibration we determined the zero point from the standard star fields observed during the night, assuming standard values for the extinction and colour coefficients, which do not add a significant uncertainty for a site like Paranal. The night was photometric.

\subsection{Spectroscopy}

Reflectance spectra of $2 \mathrm{P} /$ Encke and the NEOs were obtained using a $1.0^{\prime \prime}$ slit together with the low-resolution grism $150 \mathrm{I}+27$, that covers the full visible spectrum from $400 \mathrm{~nm}$ to $900 \mathrm{~nm}$ on the detector. For calibration purposes, spectra of solar analogue stars were taken at similar airmasses and with the instrument in the same configuration. Standard methods of spectroscopy reduction and extraction were applied: bias subtraction, flat fielding using lamp flats, wavelength calibration using arc lamp spectra, exposure time normalisation, and sky background subtraction. To remove the solar spectrum from each of the objects, we divided the object's spectra by the average of the solar analogue spectra. The object spectra were then normalised to unity at $638 \mathrm{~nm}$ (central wavelength of the $R$ Special filter).

\section{Results: $2 \mathrm{P} /$ Encke and the NEOs}

If $2 \mathrm{P} /$ Encke, the NEOs, Sutter's Mill and Maribo are parts of a same body we expect that they show, in addition to common dynamical properties, also similar spectral properties. Moreover, it is reasonable to expect that, since $2 \mathrm{P} /$ Encke shows activity in a good part of its orbit, the NEOs could show cometary activity as well. At the time of the observation, the majority of the NEOs were at an heliocentric distance where $2 \mathrm{P} /$ Encke has been shown to be active. Figure 1 shows the orbits of $2 \mathrm{P} /$ Encke and the NEOs and their location at the time of the observations.

\subsection{Photometry}

Examples of images of $2 \mathrm{P} /$ Encke and the NEOs are shown in Fig. 2.

Using aperture photometry we measured the magnitude of the object in each frame. The measured photometry was reduced to heliocentric distance $r=1 \mathrm{AU}$, geocentric distance $\Delta=1 \mathrm{AU}$, and phase angle $\alpha=0^{\circ}$ using:

$H=m(1,1,0)=m_{\text {meas }}-5 \log (r \Delta)+2.5 \log [\Phi(\alpha)]$

where $m_{\text {meas }}$ is the measured magnitude.

For the phase function correction we used a linear approximation (typically used for comets):

$2.5 \log [\Phi(\alpha)]=-\alpha \cdot \beta$

with the linear phase coefficient $\beta=0.05 \mathrm{mag} /{ }^{\circ}$ as determined for $2 \mathrm{P} /$ Encke by Fernández et al. (2000), and the IAU-adopted phase law (typically used for asteroids) (Meeus 1998):

$2.5 \log [\Phi(\alpha)]=2.5 \log \left[(1-G) \Phi_{1}+G \Phi_{2}\right]$

with

$\Phi_{1}=\exp \left[-3.33\left(\tan \frac{\alpha}{2}\right)^{0.63}\right]$
$\Phi_{2}=\exp \left[-1.87\left(\tan \frac{\alpha}{2}\right)^{1.22}\right]$

with the "slope parameter" $G=0.15$ (a typical value for asteroids). 
A\&A 584, A97 (2015)
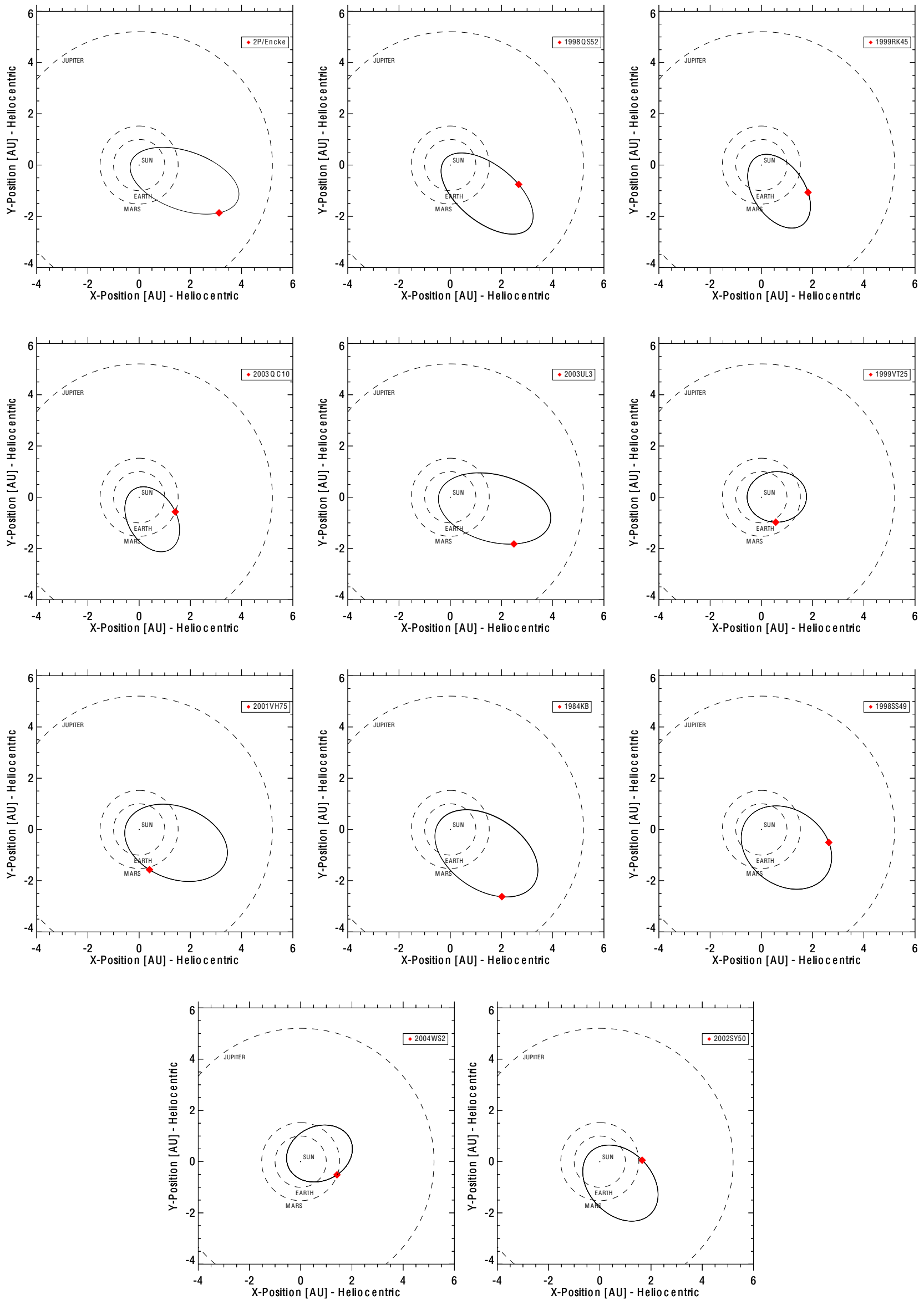

Fig. 1. Orbital position of 2P/Encke and the NEOs at the time of the observations. The orbits of the Earth, Mars, and Jupiter are displayed. 
C. Tubiana et al.: 2P/Encke, the Taurid complex NEOs and the Maribo and Sutter's Mill meteorites

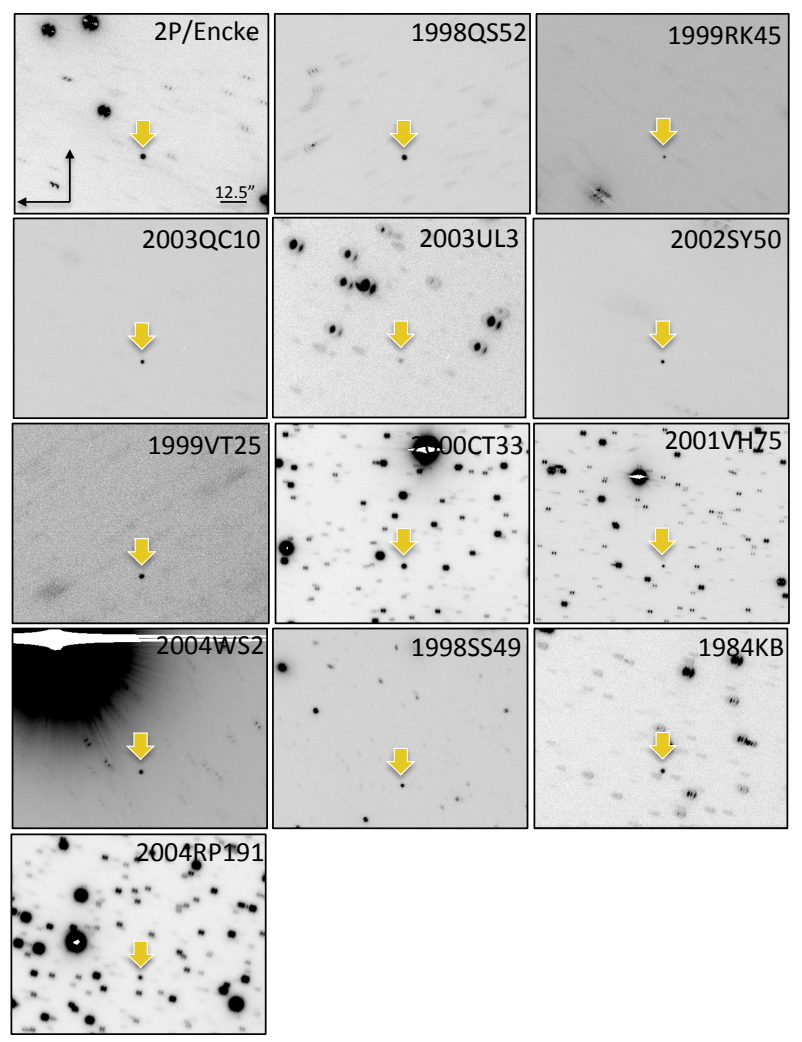

Fig. 2. Median combination of images of 2P/Encke and the NEOs. Image scale and orientation are shown. The position of the objects is marked by an arrow.

Table 3. Photometry results.

\begin{tabular}{lccc}
\hline \hline Object name & $H_{a}{ }^{a}(\mathrm{mag})$ & $H_{a}{ }^{b}(\mathrm{mag})$ & $R_{\mathrm{eff}}(\mathrm{km})$ \\
\hline $2 \mathrm{P} /$ Encke & $14.17 \pm 0.03$ & $14.34 \pm 0.03$ & $3.7^{c}, 3.4^{d}$ \\
\hline $1998 \mathrm{QS}_{52}$ & $14.03 \pm 0.03$ & $14.09 \pm 0.03$ & $3.9^{c}, 3.8^{d}$ \\
$1999 \mathrm{RK}_{45}$ & $18.77 \pm 0.05$ & $18.83 \pm 0.05$ & $0.4^{c}, 0.4^{d}$ \\
$2003 \mathrm{QC}_{10}$ & $17.61 \pm 0.03$ & $17.24 \pm 0.03$ & $0.8^{c}, 0.9^{d}$ \\
$2003 \mathrm{UL}_{3}$ & $17.46 \pm 0.08$ & $17.63 \pm 0.08$ & $0.8^{c}, 0.7^{d}$ \\
\hline $1999 \mathrm{VT}_{25}$ & $20.98 \pm 0.05$ & $20.28 \pm 0.05$ & $0.2^{c}, 0.2^{d}$ \\
$2001 \mathrm{VH}_{75}$ & $18.08 \pm 0.04$ & $17.86 \pm 0.04$ & $0.6^{c}, 0.7^{d}$ \\
$1984 \mathrm{~KB}_{1998 \mathrm{SS}_{49}}$ & $15.87 \pm 0.04$ & $16.00 \pm 0.04$ & $1.7^{c}, 1.6^{d}$ \\
$2004 \mathrm{WS}_{2}$ & $17.74 \pm 0.04$ & $15.49 \pm 0.04$ & $2.0^{c}, 2.0^{d}$ \\
$2002 \mathrm{SY}_{50}$ & $17.01 \pm 0.04$ & $17.36 \pm 0.03$ & $0.7^{c}, 0.8^{d}$ \\
\hline $2000 \mathrm{CT}_{33}$ & $14.26 \pm 0.03$ & $14.42 \pm 0.03$ & $3.5^{c}, 3.3^{d}$ \\
$2004 \mathrm{RP}_{191}$ & $16.45 \pm 0.04$ & $16.58 \pm 0.04$ & $1.3^{c}, 1.2^{d}$ \\
\hline
\end{tabular}

Notes. ${ }^{(a)}$ Average absolute magnitude of the object and associated error obtained using the IAU-adopted phase law as phase function approximation. ${ }^{(b)}$ Average absolute magnitude of the object and associated error obtained using the linear phase function approximation. ${ }^{(c)}$ Effective radius corresponding to the absolute magnitude in Col. a. ${ }^{(d)}$ Effective radius corresponding to the absolute magnitude in Col. b.

The average absolute magnitude obtained with both phase function approximations is summarised in Table 3. The corresponding effective radius of the objects (in $\mathrm{km}$ ) is calculated using (Meeus 1998):

$R_{\mathrm{eff}}=1.496 \times 10^{8} \sqrt{\frac{\left.10^{0.4\left(M_{\odot}-H\right.}\right)}{A}}$,
Table 4. Average absolute magnitude of the observed objects determined with different values of the "slope parameter".

\begin{tabular}{lccc}
\hline \hline Object name & $H_{G=0.15}(\mathrm{mag})$ & $H_{G=0.05}(\mathrm{mag})$ & $H_{G=-0.25}(\mathrm{mag})$ \\
\hline 2P/Encke & $14.17 \pm 0.03$ & 14.10 & 13.87 \\
\hline $1998 \mathrm{QS}_{52}$ & $14.03 \pm 0.03$ & 13.91 & 13.44 \\
$1999 \mathrm{RK}_{45}$ & $18.77 \pm 0.05$ & 18.65 & 18.18 \\
$2003 \mathrm{QC}_{10}$ & $17.61 \pm 0.03$ & 17.41 & 16.37 \\
$2003 \mathrm{UL}_{3}$ & $17.46 \pm 0.08$ & 17.39 & 17.17 \\
\hline $1999 \mathrm{VT}_{25}$ & $20.98 \pm 0.05$ & 20.74 & 19.25 \\
$2001 \mathrm{VH}_{75}$ & $18.08 \pm 0.04$ & 17.90 & 17.05 \\
$1984 \mathrm{~KB}_{1998}$ & $15.87 \pm 0.04$ & 15.84 & 15.75 \\
$209 \mathrm{SS}_{49}$ & $15.46 \pm 0.04$ & 15.33 & 14.81 \\
$2004 \mathrm{WS}_{2}$ & $17.74 \pm 0.03$ & 17.54 & 16.49 \\
\hline
\end{tabular}

Notes. The error associated to the average absolute magnitude, as reported in the second column, is representative of the uncertainty associated to all determinations of the average absolute magnitude.

where $A=0.04$ is the assumed geometric albedo of the object, $H$ and $M_{\odot}$ are the absolute magnitude of the object and of the Sun in the same wavelength, respectively.

If the Taurid NEOs are indeed pieces of a parent body that produced $2 \mathrm{P} /$ Encke, it is possible that their albedo is low. Primitive and low-albedo objects may have values of the "slope parameter" G much lower than the one generally assumed for asteroids. Table 4 summarises the average absolute magnitudes of the observed objects for $G=0.05$ and $G=-0.25$. The first value is representative of low-albedo objects and the second one is the slope parameter determined by Fernández et al. (2000) for $2 \mathrm{P} /$ Encke.

To investigate the possible presence of cometary activity around the nucleus of $2 \mathrm{P} /$ Encke and the NEOs, we compared the surface brightness profile of the objects with the one of a star, following the method described in Tubiana et al. (2008): we measure the profile across the width of the trailed star profile in the same image as the asteroid. All objects (including 2P/Encke) show star-like profiles (see Fig. 3), indicating that no detectable activity is present around the object.

However, 2P/Encke is brighter than expected. In fact, the effective radius that we obtained is larger then the one determined by Fernández et al. (2000). Here are a few possible explanations of this discrepancy:

1. $2 \mathrm{P} /$ Encke is weakly active at the time of our observations. No resolved coma is present around the nucleus, as shown by the radial profile (see Fig. 3), but it is possible that activity is within the seeing disc and therefore at a low level. Comparing the magnitude we observe with that found by Fernández et al. (2000), we would expect that the coma contributes $58 \%$ of the flux within the aperture.

However, it might not be reasonable to believe that about $\sim 60 \%$ of the additional light is within a coma area of $\sim 3000 \mathrm{~km}$ around the nucleus and it drops in a way that it is not detected in the more distant profile, since the dust coma intensity drops following $1 / r$, while the seeing disc is steeper. Significant activity cannot be the only source of the discrepancy.

2. Fernández et al. (2000) determined the radius of 2P/Encke using the flux corresponding to the midway between the maximum and the minimum flux of the rotational light curve. If our observation lays close to the maximum of the 

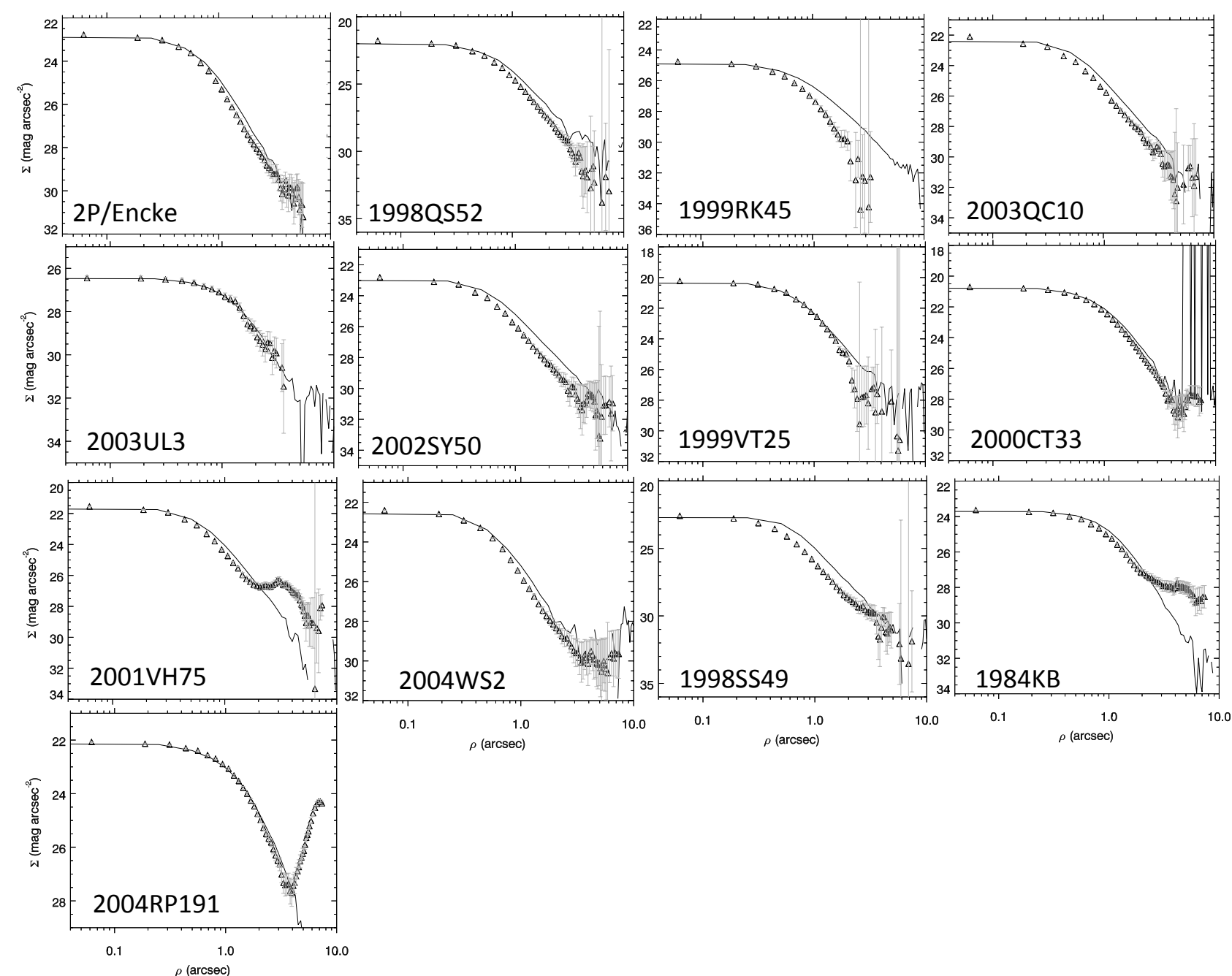

Fig. 3. Surface brightness profiles for $2 \mathrm{P} /$ Encke and the NEOs. For each object, the triangles show the profile of the object, while the solid line shows the image PSF.

rotational light curve, our flux is higher than the one measured by Fernández et al. (2000), and this could contribute to the discrepancy in the radius.

3. The set of parameters used in the thermal model used to determine the radius of $2 \mathrm{P} /$ Encke contains various assumptions, such as the beaming parameter, which are reasonable but contribute some extra uncertainty.

4. When Fernández et al. (2000) observed 2P/Encke, the comet was active. They estimated the coma contribution to the flux and they subtracted it. This determination might be source of the discrepancy.

5. The photometry of $2 \mathrm{P} /$ Encke obtained in this analysis is well in agreement with what was found by Fernández et al. (2005) for similar heliocentric distances and phase angle. This could mean that the nucleus of $2 \mathrm{P} /$ Encke is indeed larger than the determination of Fernández et al. (2000), that the phase darkening is steeper and that the radar results obtained by Harmon \& Nolan (2005) need to be re-interpreted. Another explanation could be that $2 \mathrm{P} /$ Encke has a consistent activity in the orbital arc 3.5-3.7 AU outbound, although activity cannot completely explain the discrepancy, as discussed in point 1 above.

The radial profiles of the NEOs rule out strong activity of these objects at the time of the observation, but they could still have an "Encke"-like behaviour, as described in scenario 1 above. Moreover, we have only snap-shot observations and each object was at a different location in its orbit, at different heliocentric distance. 2P/Encke, $2003 \mathrm{UL}_{3}, 1999 \mathrm{VT}_{25}, 2001 \mathrm{VH}_{75}$, $1984 \mathrm{~KB}$, and $2004 \mathrm{WS} 2$ were moving outbound while the other NEOs (1998 $\mathrm{QS}_{52}, 1999 \mathrm{RK}_{45}, 2003 \mathrm{QC}_{10}, 1998 \mathrm{SS}_{49}$, and $2002 \mathrm{SY}_{50}$ ) were in the inbound leg. Comets tend to show activity to larger distance post perihelion (Kelley et al. 2013). Heliocentric light curves of the NEOs would provide a more complete information about the activity behaviour of these objects at different distances from the Sun.

Assuming that the observed NEOs are truly inactive, a couple of objects are of comparable size as $2 \mathrm{P} /$ Encke. If they are all fragments of the same body, Encke's apparently unique continuing activity can not be explained by it being the largest fragment.

\subsection{Spectroscopy}

We obtained 3-4 low resolution spectra of each object in the wavelength range 400-900 nm. For each object, since all spectra looked very similar, we median them to increase the signalto-noise ratio. An issue with the solar analogue stars creates an artefact in the spectra around $600 \mathrm{~nm}$ and around $750 \mathrm{~nm}$, thus 
C. Tubiana et al.: 2P/Encke, the Taurid complex NEOs and the Maribo and Sutter's Mill meteorites

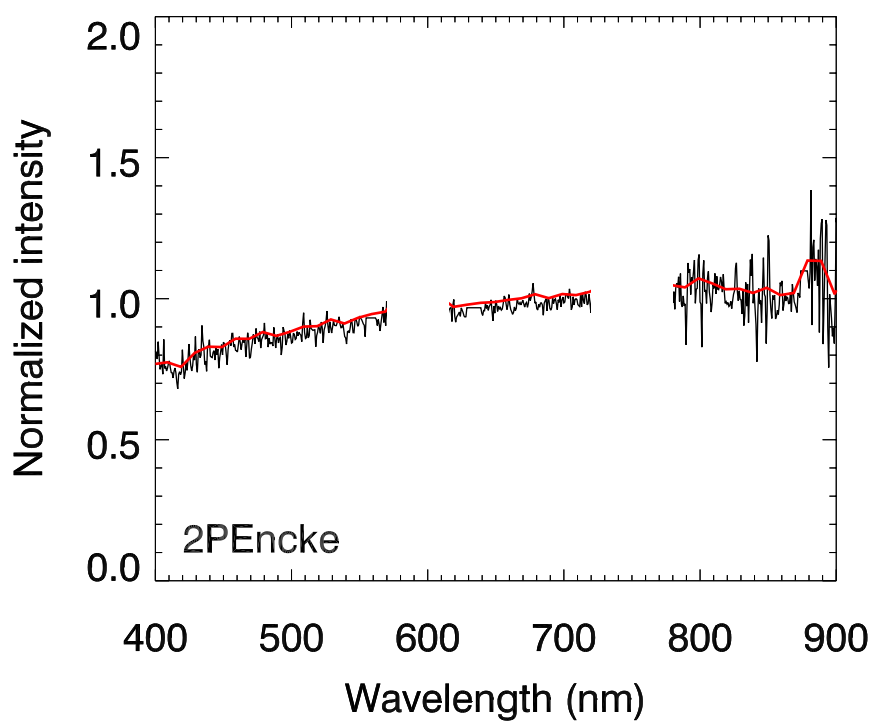

Fig. 4. Low resolution spectrum of the nucleus of $2 \mathrm{P} /$ Encke. The spectrum is normalised to unity at $638 \mathrm{~nm}$. On the original spectrum (black line), the binned one (red line) is superimposed. The gaps around $600 \mathrm{~nm}$ and $750 \mathrm{~nm}$ are areas that have been masked due to artefacts created by issues in the solar analogue star spectrum at those wavelengths. The same normalisation, binning and masking also applies to all other spectra shown in this paper (Figs. 5, 7, 8).

we masked these regions to avoid wrong interpretations of the spectra.

Figure 4 displays the low resolution spectrum of the nucleus of $2 \mathrm{P} /$ Encke. This is the first high signal-to-noise ratio visible spectrum of the bare nucleus of this comet. It is featureless and displays a reddening slope of $(7.3 \pm 0.2) \% / 100 \mathrm{~nm}$ in the wavelength range $\Delta \lambda=420-750 \mathrm{~nm}$ and $(5.0 \pm 0.2) \% / 100 \mathrm{~nm}$ in the wavelength range $\Delta \lambda=420-900 \mathrm{~nm}$. The continuum of $2 \mathrm{P} /$ Encke flattens off beyond about $800 \mathrm{~nm}$, which results in the shallower slope for the wider wavelength coverage. As FORS spectra are noisy beyond $800 \mathrm{~nm}$, we use the former range to measure the slope for all other objects.

Very few spectra of cometary nuclei exist in the literature, they are in general featureless and with a fairly constant and moderate slope in the visible wavelength range (Luu 1993; Tubiana et al. 2008, 2011). Comet 67P/ChuryumovGerasimenko has a featureless spectrum with a reddening slope of $11 \pm 2 \% / 100 \mathrm{~nm}$ in the same wavelength range, from FORS observations with an identical setup (Tubiana et al. 2011). Compared to the available spectra of other cometary nuclei, the spectrum of $2 \mathrm{P} /$ Encke is rather typical, despite $2 \mathrm{P} /$ Encke being in a very peculiar orbit.

The spectra of $1998 \mathrm{QS}_{52}, 1999 \mathrm{RK}_{45}, 2003 \mathrm{QC}_{10}$, and $2003 \mathrm{UL}_{3}$ (NEOs belonging to Group 1) are shown in Fig. 5. All spectra look featureless for $\lambda<800 \mathrm{~nm}$ and their reddening slopes are summarised in Table 5. Michelsen et al. (2006) observed a strong phase reddening in NEO spectra at $\alpha>60^{\circ}$. Our observations were performed at $\alpha \leq 53^{\circ}$, thus the phase reddening should not hinder our comparison between asteroids.

$1998 \mathrm{QS}_{52}, 1999 \mathrm{RK}_{45}$, and $2003 \mathrm{UL}_{3}$ display a slope comparable to the one of $2 \mathrm{P} /$ Encke. The spectrum of $2003 \mathrm{UL}_{3}$ is quite noisy, we do not consider the drop of flux at the redend of the spectrum as a real feature, due to the low signal-tonoise ratio in this region. The spectrum of $1998 \mathrm{QS}_{52}$ has higher signal-to-nose ratio also at long wavelengths and the drop of flux at $\lambda>800 \mathrm{~nm}$ seems real. $2003 \mathrm{QC}_{10}$, instead, has a flatter
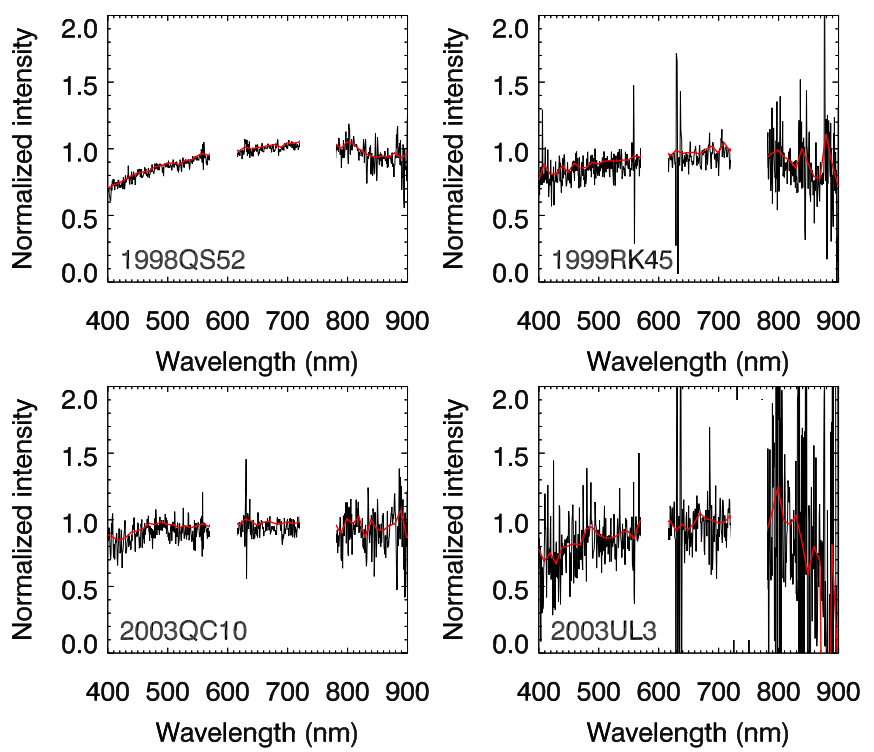

Fig. 5. Low resolution spectra of $1998 \mathrm{QS}_{52}, 1999 \mathrm{RK}_{45}, 2003 \mathrm{QC}_{10}$, and $2003 \mathrm{UL}_{3}$. The spike visible at about $650 \mathrm{~nm}$ is a skyline not completely removed during the spectra reduction.

Table 5. Reddening slopes of low resolution spectra.

\begin{tabular}{lc}
\hline \hline Object & $S^{a}(\% / 100 \mathrm{~nm})$ \\
\hline 2P/Encke & $7.3 \pm 0.2$ \\
& $5.0 \pm 0.2^{b}$ \\
\hline $1998 \mathrm{QS}_{52}$ & $8.1 \pm 0.3$ \\
$1999 \mathrm{RK}_{45}$ & $6.1 \pm 0.6$ \\
$2003 \mathrm{QC}_{10}$ & $2.7 \pm 0.4$ \\
$2003 \mathrm{UL}_{3}$ & $7 \pm 2$ \\
\hline $1999 \mathrm{VT}_{25}$ & $-0.4 \pm 0.4$ \\
$2001 \mathrm{VH}_{75}$ & $9.2 \pm 0.4$ \\
$1984 \mathrm{~KB}$ & $9.8 \pm 0.5$ \\
$1998 \mathrm{SS}_{49}$ & $9.1 \pm 0.3$ \\
$2004 \mathrm{WS}_{2}$ & $7.7 \pm 0.3$ \\
$2002 \mathrm{SY}_{50}$ & $8.1 \pm 0.3$ \\
\hline $2000 \mathrm{CT}_{33}$ & $12.9 \pm 0.3$ \\
$2004 \mathrm{RP}_{191}$ & $13.2 \pm 0.4$ \\
\hline
\end{tabular}

Notes. ${ }^{(a)}$ Reddening slope over $\lambda=420-750 \mathrm{~nm} .{ }^{(b)}$ Measured over $\lambda=420-900 \mathrm{~nm}$.

spectrum than the nucleus of $2 \mathrm{P} /$ Encke. The integration back in time of its orbit supports the hypothesis that this NEO is a member of the family, but the orbit must have evolved. Based on the assumption on common origins, the possible explanations of the different slopes are:

1. A close approach to a planet might have produced changes in the surface properties of $2003 \mathrm{QC}_{10}$ and therefore its spectral slope (DeMeo et al. 2014).

2. Heating might have changed the surface of $2003 \mathrm{QC}_{10}$. $2 \mathrm{P} /$ Encke spends more time close to the Sun than $67 \mathrm{P} /$ Churyumov-Gerasimenko. Comparing the spectrum of $2 \mathrm{P} /$ Encke with the one of $67 \mathrm{P} / \mathrm{Churyumov}-$ Gerasimenko, the first is slightly shallower $(\sim 7 \% / 100 \mathrm{~nm}$ for $2 \mathrm{P} /$ Encke and $\sim 11 \% / 100 \mathrm{~nm}$ for $67 \mathrm{P} /$ Churyumov-Gerasimenko). The orbit of $2003 \mathrm{QC}_{10}$ experiences greater average insolation over its orbit than $2 \mathrm{P} /$ Encke, thus higher heating might be the explanation of a shallower spectral slope. Hainaut et al. (2012) found that minor bodies in the outer solar system show a 


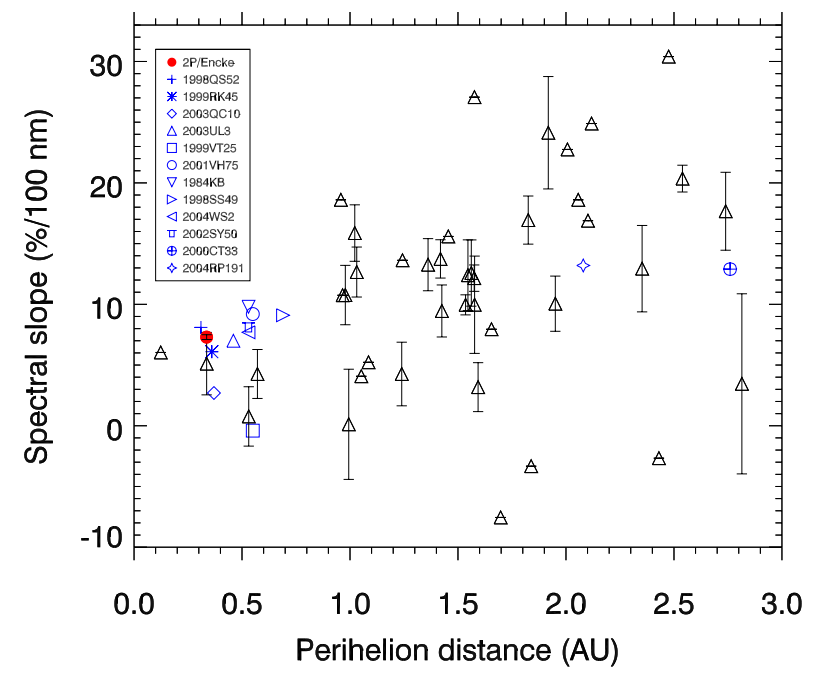

Fig. 6. Spectral slope versus perihelion distance, where a weak trend of steeper slopes at larger distances is found. Data taken from the MBOSS colour database (Hainaut et al. 2012). The red filled circle and the blue symbols correspond to the spectral slope of the spectrum of 2P/Encke and the observed asteroids, respectively, determined in this work (wavelength range $\Delta \lambda=420-750 \mathrm{~nm}$ ).

weak trend of steeper slopes at larger distances, as shown in Fig. 6.

3. Another possibility is that the giant comet collided with another object, causing an initial fragmentation so that $2003 \mathrm{QC}_{10}$ is essentially from another body (the remains of the impactor). This is a possible explanation of why 2003 QC $_{10}$ is different from Encke and the other 3 asteroids belonging to Group 1, although it seems improbable.

Figure 7 displays the spectra of the NEOs belonging to Group 2. Also in this case the spectra do not show any absorption or emission feature for $\lambda<800 \mathrm{~nm} .1984 \mathrm{~KB}, 1998 \mathrm{SS}_{49}, 2001 \mathrm{VH}_{75}$, $2002 \mathrm{SY}_{50}$, and $2004 \mathrm{WS}_{2}$ show the silicate absorption at the red-end of the spectrum. While $1999 \mathrm{VT}_{25}$ is flat, the other objects display a moderate reddening, in the selected wavelength range, comparable with the one of $2 \mathrm{P} /$ Encke. Looking at the orbital plots (Fig. 1), the orbit of $1999 \mathrm{VT}_{25}$ is quite different from the one of $2 \mathrm{P} /$ Encke. Even though this object was considered a possible family candidate, it can instead be an interloper.

For completeness, Fig. 8 show the spectra of $2000 \mathrm{CT}_{33}$ and $2004 \mathrm{RP}_{191}$, two unrelated asteroids observed on the same night. These spectra are featureless at $\lambda<800 \mathrm{~nm}$ and they are redder than the one of $2 \mathrm{P} /$ Encke. The latter spectrum also displays a decrease in flux towards the red-end of the spectrum, typical of S-type asteroids. Since these two object are not linked with 2P/Encke, we do not expect them to show spectral similarities.

In conclusion, the spectra of $2 \mathrm{P} /$ Encke and of all the selected NEOs are featureless for $\lambda<800 \mathrm{~nm} .1998 \mathrm{QS}_{52}$ and all NEOs belonging to Group 2 (except for $1999 \mathrm{VT}_{25}$ ) show the silicate absorption at the red-end of the spectrum. Except for $2003 \mathrm{QC}_{10}$ and $1999 \mathrm{VT}_{25}$ that have a flatter spectrum, the other objects belonging to Group 1 and Group 2 show a moderate reddening slope at $\lambda<800 \mathrm{~nm}$, compatible with the one of $2 \mathrm{P} /$ Encke, as it can be seen in Fig. 9.

It is difficult to draw a firm conclusion if those NEOs and $2 \mathrm{P} /$ Encke are linked just based on their spectral properties since the only available parameter is the spectral slope. If we believe that the spectral slope per se is indicative of belonging to the same family, then $2 \mathrm{P} /$ Encke and $1998 \mathrm{QS}_{52}, 1999 \mathrm{RK}_{45}$,
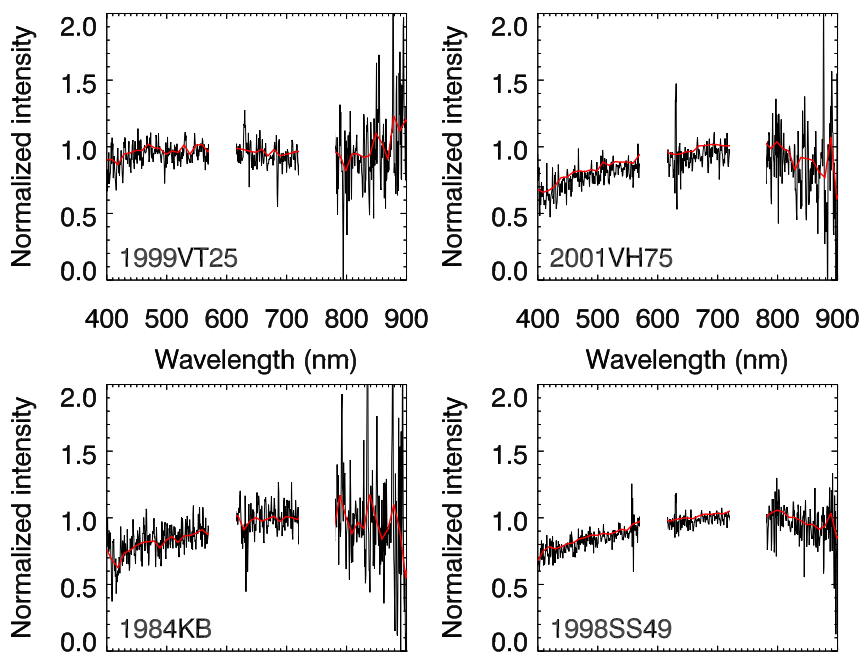

400500600700800900 Wavelength $(\mathrm{nm})$

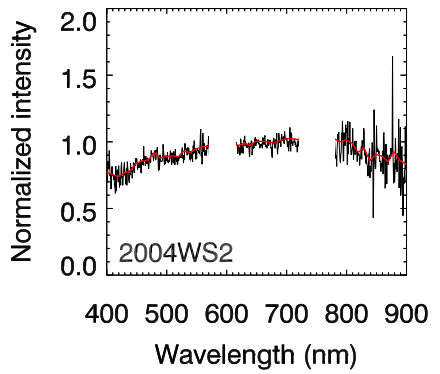

400500600700800900 Wavelength $(\mathrm{nm})$

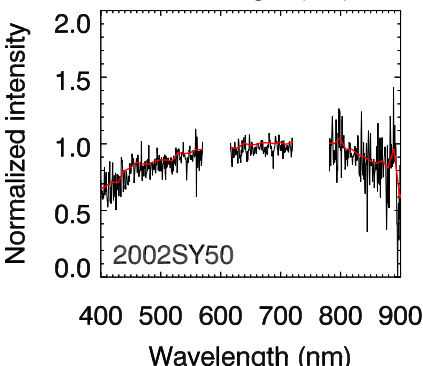

Fig. 7. Low resolution spectra of $1999 \mathrm{VT}_{25}, 2001 \mathrm{VH}_{75}, 1984 \mathrm{~KB}$, $1998 \mathrm{SS}_{49}, 2004 \mathrm{WS}_{2}$, and $2002 \mathrm{SY}_{50}$.
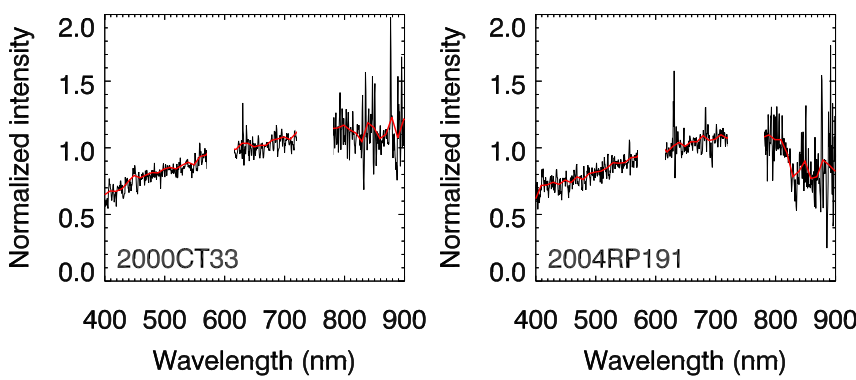

Fig. 8. Low resolution spectra of $2000 \mathrm{CT}_{33}$ and $2004 \mathrm{RP}_{191}$.

$2003 \mathrm{UL}_{3}, 2001 \mathrm{VH}_{75}, 1984 \mathrm{~KB}, 1998 \mathrm{SS}_{49}, 2004 \mathrm{WS}_{2}$, and $2002 \mathrm{SY}_{50}$ might be related. The absence of absorption features is a common characteristic to other comets as well, thus it cannot be used to enforce the link between $2 \mathrm{P} /$ Encke and the selected NEOs, but does at least demonstrate that Taurid complex NEOs are compatible with a cometary source based on spectral slopes.

However, including the information contained in the noisier part of the spectrum in the $800>\lambda>900 \mathrm{~nm}$ range shows that the spectral slope gives only part of the picture. In this region many of the NEOs show evidence of a weak absorption feature, most likely due to silicates, which is not seen in cometary spectra.

\subsection{NEOs classification based on the shape of their spectrum}

We have classified our targets according to the Bus-DeMeo taxonomy (DeMeo et al. 2009), which uses both optical and 
C. Tubiana et al.: 2P/Encke, the Taurid complex NEOs and the Maribo and Sutter's Mill meteorites

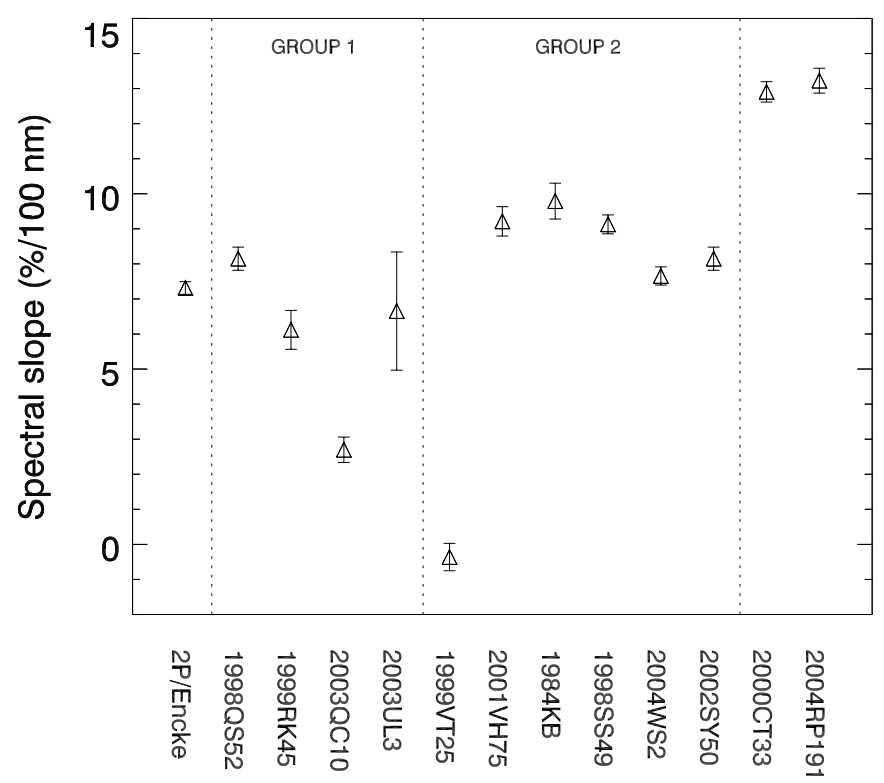

Fig. 9. Spectral slope of $2 \mathrm{P} /$ Encke and the selected NEOs in the wavelength range $\Delta \lambda=420-750 \mathrm{~nm}$, as summarised in Table 5 .

near-IR spectra to identify 24 spectral classes existing within the asteroid population. This classification scheme is an update of the original Bus taxonomy that was based on optical spectra only (Bus 1999). Although we only have optical data, DeMeo et al. (2009) show a close correspondence between this taxonomy with the previous optical-based system, therefore we have chosen the newer scheme as the most complete spectral classification system to date. For each spectrum we calculated the mean reflectance over $50 \mathrm{~nm}$ bins at each of the standard wavelengths in the taxonomic scheme (450 nm to $900 \mathrm{~nm})$. We then simply calculated the $\chi^{2}$ value between the target spectrum and the 24 spectral classes to identify the best fit, checking by eye that the identified spectral matches looked reasonable. The resulting spectral classifications are given in Table 6. Where it was impossible to assign a unique classification to an object, all likely spectral classes are listed. In assigning the listed classifications we ignored the bin centres at $600 \mathrm{~nm}$ and $750 \mathrm{~nm}$ due to problems with the reflectance spectra in those regions, but noted that including them made no significant difference. Two of our targets have been spectrally classified previously. $1998 \mathrm{QS}_{52}$ was observed by Binzel et al. (2004) in their large study of the NEO population, and found to be Sq-class. Our classification of $\mathrm{Sq} / \mathrm{Sr}$ is consistent with theirs. Popescu et al. (2011) also find this NEO to be Sr class based on near infrared data. Asteroid $1984 \mathrm{~KB}$ was classified as S-class by Bell et al. (1988) and Sq by Popescu et al. (2014). Again, our classification of K/Sq places this object firmly within the S-complex of asteroids, and is consistent with the previous research. A recent paper by DeMeo et al. (2014) includes classifications for $1998 \mathrm{SS}_{49}$ and $2002 \mathrm{SY}_{50}$, which are also found to be $S$-types based on near infrared data.

Going by the groups listed in Table 1, 2P/Encke is identified as an X-class object in this scheme, with the best match being $\mathrm{Xe}$. An X-class classification is no surprise, as the X-complex comprises of E, M and P-class asteroids, all of which show a moderate red slope with no spectral features that can only be differentiated by albedo knowledge. This exactly describes comet nuclei with moderate red spectral slopes. Three of the Group 1 Taurids are clearly silicate, although the $\mathrm{S} / \mathrm{N}$ in the spectrum of $2003 \mathrm{UL}_{3}$ is too low for a clear classification. $2003 \mathrm{QC}_{10}$ is likely
Table 6. Spectral type of the objects.

\begin{tabular}{lc}
\hline \hline Object & Spectral type \\
\hline 2P/Encke & $\mathrm{Xe}$ \\
\hline Group 1 Taurids: & \\
\hline $1998 \mathrm{QS}_{52}$ & $\mathrm{Sq} / \mathrm{Sr}$ \\
$1999 \mathrm{RK}_{45}$ & $\mathrm{Q}$ \\
$2003 \mathrm{QC}_{10}$ & $\mathrm{C} / \mathrm{Xn}$ \\
$2003 \mathrm{UL}_{3}$ & $\mathrm{~V} / \mathrm{O} / \mathrm{Q}$ \\
\hline Group 2 Taurids: & \\
\hline $1984 \mathrm{~KB}$ & $\mathrm{~K} / \mathrm{Sq}$ \\
$1998 \mathrm{SS}_{49}$ & $\mathrm{Sq} / \mathrm{Sr}$ \\
$1999 \mathrm{VT}_{25}$ & $\mathrm{C}$ \\
$2001 \mathrm{VH}_{75}$ & $\mathrm{Q}$ \\
$2002 \mathrm{SY}_{50}$ & $\mathrm{Q}$ \\
$2004 \mathrm{WS}_{2}$ & $\mathrm{Q}$ \\
\hline Non-Taurid asteroids: & \\
$2000 \mathrm{CT}_{33}$ & $\mathrm{~L} / \mathrm{D}$ \\
$2004 \mathrm{RP}_{191}$ & $\mathrm{R} / \mathrm{Sa}$ \\
\hline
\end{tabular}

a C-type asteroid; the formal fitting also gives $\mathrm{Xn}$ as a possible classification, but this relies on the existence of a Nysa-like absorption feature at $900 \mathrm{~nm}$ which is in the region of low $\mathrm{S} / \mathrm{N}$ in our spectra. Similarly, all but one of the Group 2 Taurids are also silicate. We could not distinguish between the $\mathrm{Cb} / \mathrm{Ch} / \mathrm{Cgh} / \mathrm{C}$ classifications for $1999 \mathrm{VT}_{25}$, so we simply adopt C. Finally, we list for completeness the spectral classes of the two non-Taurid NEOs.

Based on the spectral classification, none of the Taurid NEOs is similar to $2 \mathrm{P} /$ Encke. Apart from $2003 \mathrm{QC}_{10}$ and $1999 \mathrm{VT}_{25}$ that are C-type, all the NEOs are silicate types. If we also consider the recent results from Popescu et al. (2014) we see a strong preference for S-complex asteroids in Taurid-like orbits. Two further objects from their sample are part of our "Group 1" list, with the most secure association with the Taurids: (4183) Cuno and (4486) Mithra, found to be Q and Sq type respectively. Two of their other targets [(2201) Oljato and (5143) Heracles] would fit into our Group 2, and are also Q or Sq types, while they only identified one C-type, (269690) $1996 \mathrm{RG}_{3}$, which was furthest from the centre of the Taurid cloud by their definition, and also fits into our Group 2. This suggests that these objects are not unusual for near-Earth and inner main belt asteroids, where S-types dominate, and do not require a large split comet to explain their origin.

\section{Results: 2P/Encke and CM chondrites}

The next step in the analysis was the comparison between the spectra of the nucleus of $2 \mathrm{P} /$ Encke and the NEOs and the ones of CM chondrites, the class to which Maribo and Sutter's Mill belong.

All the spectra we have obtained are featureless below $800 \mathrm{~nm}$ and relatively flat (see spectral slopes in Table 5). A tentative classification based on the shape of the spectra is described in Sect. 3.3. The spectrum of $2 \mathrm{P} /$ Encke resembles one of the primitive asteroids, which are believed to be associated with carbonaceous chondrites. A link between Encke and the CM chondrites, including the Maribo and Sutter's Mill meteorites, is therefore possible.

Although Maribo is too small to have a reflectance spectrum measured (a destructive process), a sufficient number of samples were collected of Sutter's Mill, and a spectrum covering the $0.3-5 \mu \mathrm{m}$ range is available (Jenniskens et al. 2012a, their 
Fig. S8). CM chondrites are carbonaceous chondrites from a parent body that was originally rich in volatiles including liquid water and organics. Aqueous alteration of metal and silicate phases resulted in the formation of phyllosilicates and iron alteration minerals. The altered phases are seen as absorption features in reflected light at $700 \mathrm{~nm}$ and $3 \mu \mathrm{m}$ (Rivkin et al. 2002). Since our observations cover the wavelength range 400-900 $\mathrm{nm}$, we should therefore be able to observe the $700 \mathrm{~nm}$, but not the $3 \mu \mathrm{m}$ feature.

As can be seen in Figs. 4, 5, and 7 none of the observed objects show signs of an absorption feature at $700 \mathrm{~nm}$. Therefore our observations do not provide a direct link between any of the observed NEOs and CM chondrites. The remaining question is if the data rule out that the CM chondrites could be associated with the observed objects.

The fact that 2P/Encke's surface spectrum does not match that of a carbonaceous chondrite does not rule out that $2 \mathrm{P} /$ Encke could be the parent body of Maribo and/or Sutter's Mill. It is important to remember that $\mathrm{CM}$ chondrites have highly variable spectral properties and that comet surfaces undergo significant changes due to Solar heating. The $700 \mathrm{~nm}$ features is caused by hydrated minerals which disappear if the material is moderately heated (Hiroi et al. 1996). It is therefore possible that the surface spectra of comets and primitive asteroids do not give a true representation of the interior composition, which is the volume sampled when looking at meteorite sample.

\section{Summary and conclusions}

We analysed $R$ filter images and low resolution spectra of 2P/Encke and 10 NEOs obtained at the ESO-VLT using FORS on August 2, 2011.

1. The comparison of the surface brightness profile of the objects with the one of a star indicates that no detectable activity is present.

2. The spectra of $2 \mathrm{P} /$ Encke and of the NEOs are featureless for $\lambda<800 \mathrm{~nm}$. $1998 \mathrm{QS}_{52}$ and all NEOs belonging to Group 2 (except for $1999 \mathrm{VT}_{25}$ ) show the silicate absorption at the red end of the spectrum. With the exception of $2003 \mathrm{QC}_{10}$ and $1999 \mathrm{VT}_{25}$ that show flatter spectra, the other objects belonging to Group 1 and 2 show a moderate reddening slope $(\sim 6-10 \% / 100 \mathrm{~nm})$, compatible with the one of $2 \mathrm{P} /$ Encke. It is however difficult to draw a firm conclusion if $2 \mathrm{P} /$ Encke and the selected NEOs are linked just based on their spectral properties, since the only available parameter is the spectral slope. The absence of absorption features at $\lambda<800 \mathrm{~nm}$ is a common characteristic to other comets as well, thus cannot be used to enforce the link between 2P/Encke and the observed NEOs.

3. The absence of the $700 \mathrm{~nm}$ absorption feature in the spectra of 2P/Encke and the selected NEOs doesn't allow us to establish a direct link between $2 \mathrm{P} /$ Encke, the observed NEOs and $\mathrm{CM}$ chondrites.

4. The spectral classification of the objects shows that none of the Taurid NEOs is similar to $2 \mathrm{P} /$ Encke when the low $\mathrm{S} / \mathrm{N}$ range $(\lambda>800 \mathrm{~nm})$ is taken into account. The best fit taxonomic Group for most of the NEOs is the S-complex, while Encke doesn't show any evidence for absorption at $\lambda>800 \mathrm{~nm}$, and is best fit by the Xe-type template.

5. The difference between the spectrum of 2P/Encke and CM chondrites are due to the presence of hydrated minerals in
CM chondrites. A connection between 2P/Encke remains ambiguous as we do not know if $2 \mathrm{P} /$ Encke's surface has been dehydrated due Solar heating.

We conclude that there is no evidence that NEOs with Taurid-complex orbits exhibit any evidence of a compositional association with $2 \mathrm{P} /$ Encke, and that the presence of potentially unrelated bodies in these orbits means that it is still impossible to choose between a cometary or asteroidal source for the Taurid CM chondrite meteorites. If, instead, we assume that the whole Taurid-complex does have a single parent body, this (presumably large) object must have contained significant inhomogeneity, to produce some cometary and some S-type fragments.

Acknowledgements. C.S. received funding from the European Union Seventh Framework Programme (FP7/2007-2013) under grant agreement No. 268421 H.H. received funding from the Danish National Research Foundation. We thank the anonymous referee for helpful comments that improved this paper.

\section{References}

Appenzeller, I., Fricke, K., Fürtig, W., et al. 1998, The Messenger, 94, 1 Asher, D. J., Clube, S. V. M., \& Steel, D. I. 1993, MNRAS, 264, 93 Bell, J. F., Hawke, B. R., \& Brown, R. H. 1988, Icarus, 73, 482

Binzel, R. P., Rivkin, A. S., Stuart, J. S., et al. 2004, Icarus, 170, 259 Böhnhardt, H., Tozzi, G. P., Bagnulo, S., et al. 2008, A\&A, 489, 1337 Bus, S. J. 1999, Ph.D. Thesis, Massachusetts Institute of Technology Clube, S. V. M., \& Napier, W. M. 1984, MNRAS, 211, 953

DeMeo, F. E., Binzel, R. P., Slivan, S. M., \& Bus, S. J. 2009, Icarus, 202, 160 DeMeo, F. E., Binzel, R. P., \& Lockhart, M. 2014, Icarus, 227, 112

Fernández, Y. R., Lisse, C. M., Käufl, H. U., et al. 2000, Icarus, 147, 145 Fernández, Y. R., Lowry, S. C., Weissman, P. R., et al. 2005, Icarus, 175, 194 Haack, H., Michelsen, R., Stober, G., et al. 2011, Meteorit. Planet. Sci. Suppl., 74,5271

Haack, H., Grau, T., Bischoff, A., et al. 2012, Meteorit. Planet. Sci., 47, 30

Hainaut, O. R., Boehnhardt, H., \& Protopapa, S. 2012, A\&A, 546, A115

Harmon, J. K., \& Nolan, M. C. 2005, Icarus, 176, 175

Hiroi, T., Zolensky, M. E., Pieters, C. M., \& Lipschutz, M. E. 1996, Meteorit. Planet. Sci., 31, 321

Hsieh, H. H., \& Jewitt, D. 2006, Science, 312, 561

Jenniskens, P., Fries, M. D., Yin, Q.-Z., et al. 2012a, Science, 338, 1583

Jenniskens, P., Girten, B., Sears, D., et al. 2012b, Meteorit. Planet. Sci. Suppl., 75,5376

Jewitt, D. 2012, AJ, 143, 66

Jewitt, D. C. 2002, AJ, 123, 1039

Jopek, T. J., \& Williams, I. P. 2013, MNRAS, 430, 2377

Kelley, M. S., Fernández, Y. R., Licandro, J., et al. 2013, Icarus, 225, 475

Lamy, P., \& Toth, I. 2009, Icarus, 201, 674

Lamy, P. L., Toth, I., Fernandez, Y. R., \& Weaver, H. A. 2004, in The sizes, shapes, albedos, and colors of cometary nuclei (Tucson: University of Arizona Press), 223

Levison, H. F., Terrell, D., Wiegert, P. A., Dones, L., \& Duncan, M. J. 2006 Icarus, 182, 161

Lodders, K., \& Osborne, R. 1999, Space Sci. Rev., 90, 289

Luu, J., \& Jewitt, D. 1990, Icarus, 86, 69

Luu, J. X. 1993, Icarus, 104, 138

Meeus, J. 1998, Astronomical algorithms (Willmann-Bell Inc.)

Michelsen, R., Nathues, A., \& Lagerkvist, C.-I. 2006, A\&A, 451, 331

Porubčan, V., Williams, I. P., \& Kornoš, L. 2004, Earth Moon and Planets, 95, 697

Porubčan, V., Kornoš, L., \& Williams, I. P. 2006, Contributions of the Astronomical Observatory Skalnate Pleso, 36, 103

Popescu, M., Birlan, M., Binzel, R., et al. 2011, A\&A, 535, A15

Popescu, M., Birlan, M., Nedelcu, D. A., Vaubaillon, J., \& Cristescu, C. P. 2014, A\&A, 572, A106

Rivkin, A. S., Howell, E. S., Vilas, F., \& Lebofsky, L. A. 2002, Asteroids III, 235

Snodgrass, C., Lowry, S. C., \& Fitzsimmons, A. 2006, MNRAS, 373, 1590

Tubiana, C., Barrera, L., Drahus, M., \& Böhnhardt, H. 2008, A\&A, 490, 377

Tubiana, C., Böhnhardt, H., Agarwal, J., et al. 2011, A\&A, 527, A113

Whipple, F. L. 1940, The Scientific Monthly, 51, 579 